\title{
Prevalence of Baker's cyst in patients with knee pain: an ultrasonographic study
}

\author{
V. Picerno, G. Filippou, I. Bertoldi, A. Adinolfi, V. Di Sabatino, \\ M. Galeazzi, B. Frediani \\ Department of Medical Sciences, Surgery and Neurosciences, Rheumatology Unit, \\ University of Siena, Italy
}

\section{SUMMARY}

The objectives of this study are to investigate the prevalence of Baker's cyst (BC) in patients with knee pain, and to assess the correlation between $\mathrm{BC}$ and severity of osteophytes and joint effusion.

A retrospective study was conducted on a group of patients with knee pain referred to our outpatient clinic for ultrasonography of the knee between January 2010 and February 2011. Patients underwent an ultrasonographic exam of the knees to assess the presence of marginal femorotibial osteophytosis, joint effusion and BC. A dichotomous score was assigned to each item (1 present, 0 absent) and severity of US signs of osteoarthritis and joint effusion were also graded semiquantitatively. Collected data were processed using logistic regression analysis to evaluate the correlation between degree of osteophytosis and joint effusion and BC. Patients affected by inflammatory joint conditions or with history of joint surgery or recent trauma were excluded.

A total of 399 patients with knee pain were studied ( 299 women), in the age range 18-89 years (mean 56.2, SD 16.3 years). 293 patients (73.4\%) showed sonographic features of osteoarthritis and 251 (62.9\%) joint effusion. $\mathrm{BC}$ was found in 102 patients (25.8\%) together with a positive association with sonographic features of osteoarthritis and joint effusion.

Our data show a prevalence of $\mathrm{BC}$ of $25.8 \%$ in a population of patients with knee pain, and suggest that $\mathrm{BC}$ is positively related to osteoarthritis and joint effusion. Ultrasonographic examination of knee is worthwhile in patients with painful osteoarthritis or evidence of effusion.

Key words: Baker's cyst, gonalgia, osteoarthritis, joint effusion, ultrasonography.

Reumatismo, 2013; 65 (6): 264-270

\section{INTRODUCTION}

aker's cyst $(1,2)(B C)$ is a buildup of fluid in the bursa between the tendons of the gastrocnemius and semimembranous muscles, which often communicates with the joint space. Its development is therefore associated with the passage of joint fluid, and may occur in various diseases, especially when there is joint effusion (1, $2)$. Although clinical symptoms may include pain and swelling, especially during maximum flexion or extension of the knee, $\mathrm{BC}$ may also be asymptomatic and pass undetected during clinical examination. Detection is important because rupture of an undiagnosed cyst may cause acute inflammation and loss of function that is difficult to distinguish from thrombophlebitis, which calls for quite different treatment $(1$,
2). Ultrasonography has proved useful and simple for detecting BC (3-7), which may be treated by aspiration, examined by arthroscopy, or monitored in time.

The aim of the present study was to assess the prevalence of Baker's cyst in patients with painful osteoarthritis of the knee, established on the basis of ultrasonographic evidence of popliteal effusion, and to evaluate the correlation between $\mathrm{BC}$, severity of osteoarthritis and degree of joint effusion.

\section{MATERIALS AND METHODS}

A retrospective study was conducted on a group of patients with knee pain referred to our outpatient clinic for ultrasonography of the knee between January 2010 and Febru- 
ary 2011. Both female and male patients, aged older than 18 and presenting knee pain were included.

Patients affected by rheumatoid arthritis or other inflammatory conditions or with history of joint surgery or recent trauma were excluded.

All ultrasound examinations were performed by an operator with a 10 year experience in musculoskeletal ultrasonography, using linear 5-13 MHz probes (Esaote Technos or Esaote MyLab70 scanners, Genoa, Italy). The following parameters were considered: signs of osteoarthritis of the knee, namely marginal femorotibial osteophytosis, joint effusion and BC. A dichotomous score was assigned to each item (1 present, 0 absent).

Severity of osteophytosis and degree of joint effusion were also graded semiquantitatively from 0 to 3 . Images were obtained according to codified European guidelines for joint study by ultrasonography (8): with patient in supine position and extended knee to evaluate the presence of joint effusion (on the suprapatellar and parapatellar pouch) and marginal osteophytosis; with posterior longitudinal and transverse scans (patient in prone position) between the medial head of gastrocnemius and semimembranosus muscles to investigate the presence of BC.

Osteophytes were defined as irregularities in joint profiles due to cortical protrusions at the lateral and medial margins of the femoro-tibial joint, producing a posterior acoustic shadow $(9,10)$.
The presence and degree of osteophytosis was used as criterion for ultrasonographic diagnosis of osteoarthritis of the knee and to assign a semiquantitative score (minor, moderate and advanced osteoarthritis) on the basis of the entity and the extension of irregularities of the bone profile (Fig. 1). Osteophytosis of femuro-tibial joint is considered an early and specific radiographic sign of knee osteoarthritis (11) and it is easily detected also with ultrasonography, unlike other radiographic diagnostic features of OA such as the joint space narrowing of the femuro-tibial joint. For this reason, in the absence of well defined and validated ultrasonographic score for osteoarthritis, we considered a scoring system based on osteophytosis, in analogy with the radiographic reference scores (3).

Joint effusion was identified as a hypoechogenic or anechoic distension of the joint space, displaceable and compressible, as for OMERACT definition (12), and measured in the suprapatellar recess. It was scored 0/absent when $<2 \mathrm{~mm}$ thick, $1 /$ minor $2-5 \mathrm{~mm}, 2 /$ moderate $5-10 \mathrm{~mm}$ and $3 /$ severe $>10 \mathrm{~mm}$, as in previous observations $(4,13)$.

Baker's cyst was recognized as a hypoechogenic cystic formation between the tendons of the medial head of gastrocnemius and semimembranous muscles (14) (Fig. 2).

Informed consent was obtained from each patient.

The data was processed using logistic regression analysis and SPSS version 12 software.
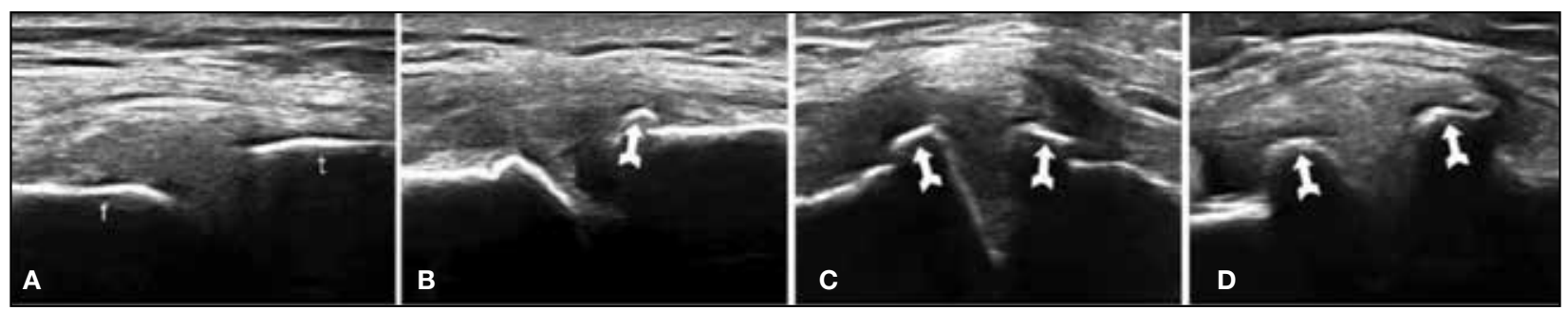

Figure 1 - Sequence of ultrasonographic images of the medial compartment of the femorotibial joint used to classify the severity of osteoarthritis (f: femur, t: tibia). a) Normal joint (grade 0); b) initial irregularity of medial femoral condyle and tibial bone profile due to minor osteophytosis, more evident on the tibia (arrow) (grade 1); c) moderate osteophytosis of the tibial and femoral surfaces with widening of joint profiles, reduction of joint space and mild extraflexion of the meniscus (grade 2); d) gross alteration of joint bone profile due to large osteophytes (arrows) and severe reduction of joint space with concomitant structural irregularity and almost complete protrusion of meniscus. 


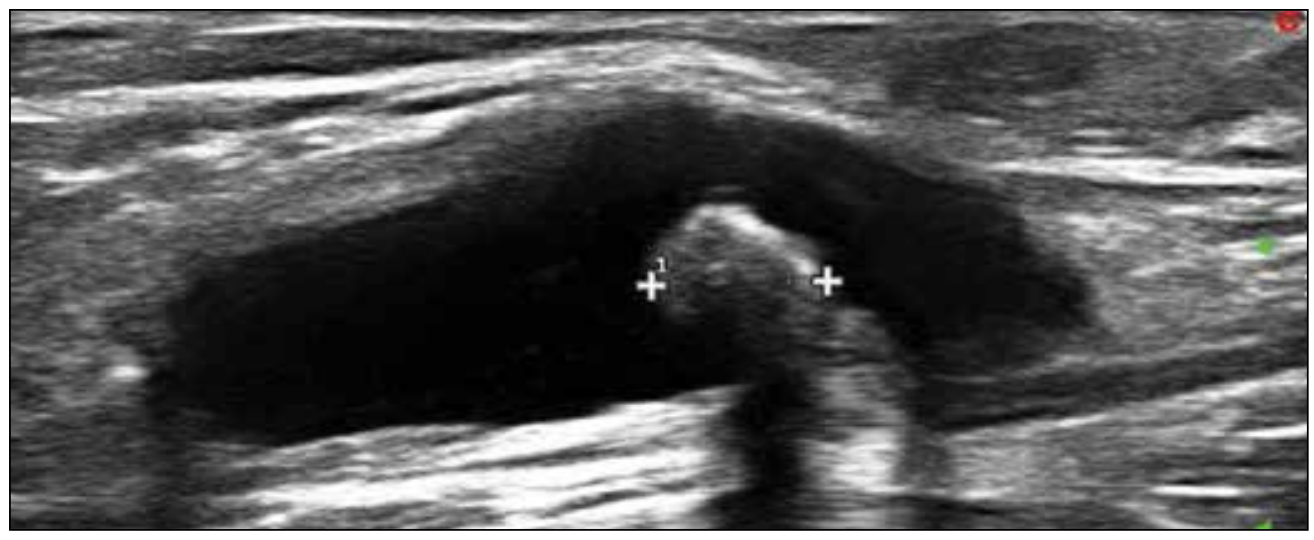

Figure 2 - Ultrasound image of Baker's cyst. The cyst is oval and located deep in the gastrocnemius and semimembranosus muscles. Distension of the bursa with fluid results in anechogenic appearance and thickening of the posterior wall. A calcification inside the cyst (indicated by caliper) appears as a gross hyperechogenic mass with posterior shadow cone.

\section{RESULTS}

The study sample consisted of 399 patients ( 299 women, 100 men) in the age range 18 89 years (mean 56.2, SD 16.3 years). 293 patients $(73.43 \%)$ showed US signs of osteophytosis of the knee, 251 (62.9\%) joint effusion and $102(25.56 \%)$ BC. $99(33.8 \%)$ patients with osteophytosis also had BC. Only three patients had BC without US signs of osteoarthritis. When patients were stratified according to severity of osteoar- thritis, BC was found in $25.45 \%(42 / 165)$ of those with slight, $47.88 \%$ (34/71) of those with moderate and $40.35 \%$ (23/57) of those with severe US signs of osteoarthritis (Fig. 3).

Of the 251 patients with joint effusion, $33.8 \%$ (85) showed evidence of $\mathrm{BC}$; $17 / 102$ BCs $(16.7 \%)$ were not associated with joint effusion. When patients were stratified according to degree of effusion, $31.93 \%(61 / 191)$ of patients with slight effusion showed BC, whereas the corre-

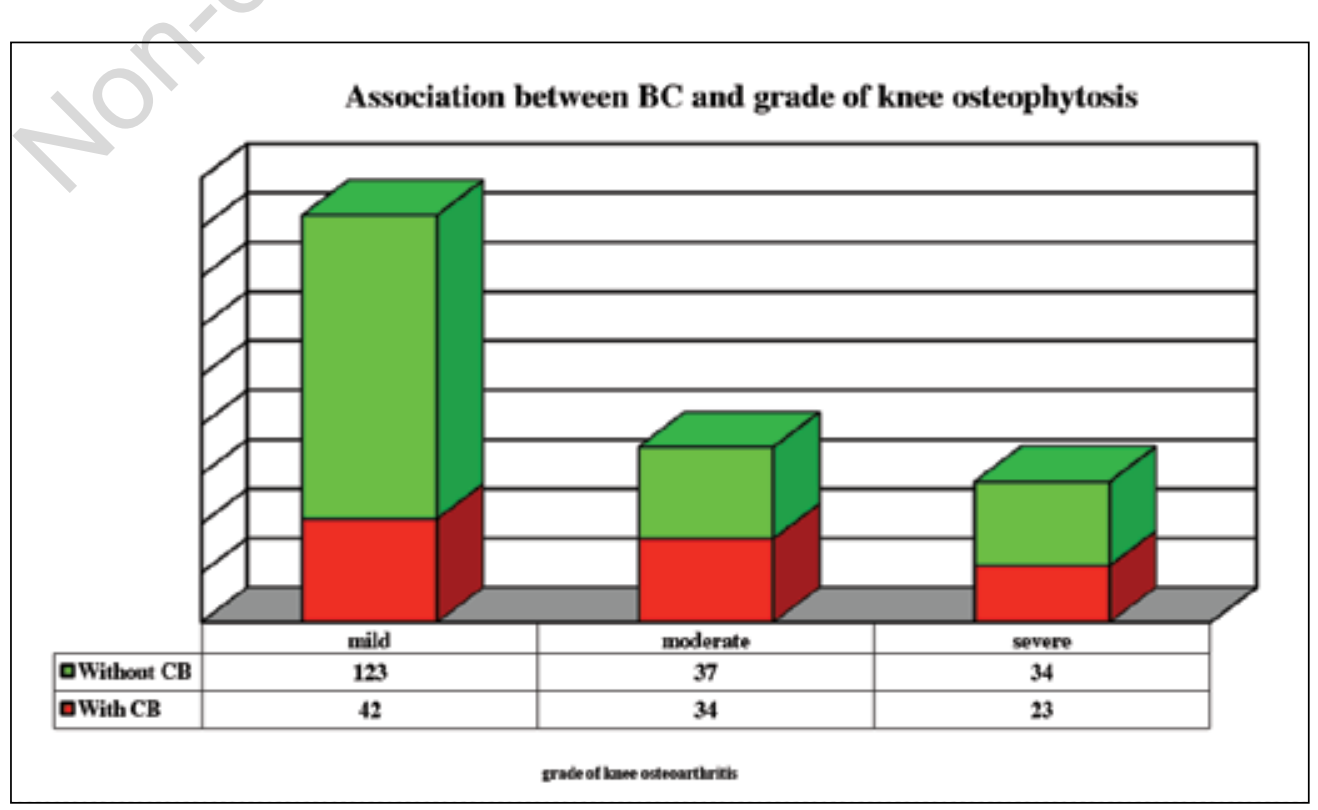

Figure 3 - Prevalence of Baker's cysts in relation to severity of osteophytosis. 


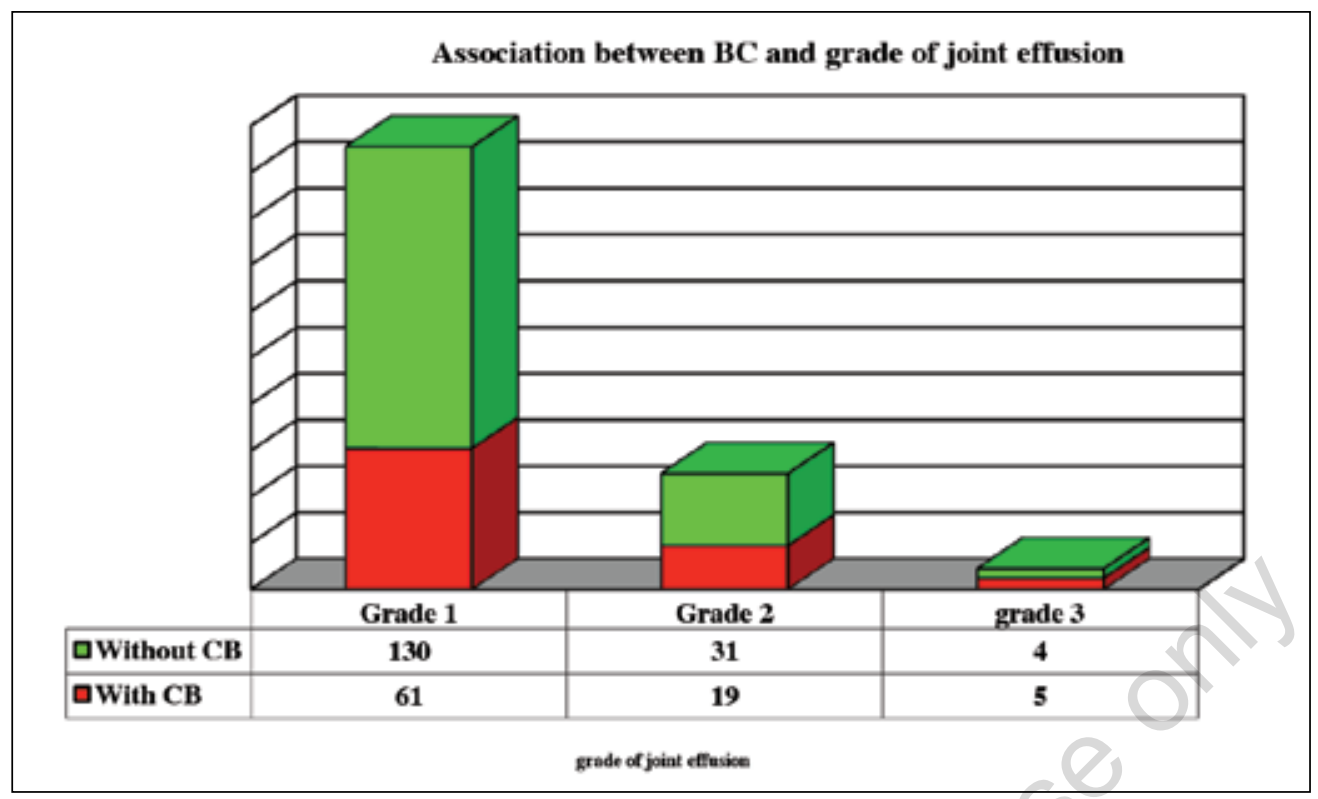

Figure 4 - Prevalence of Baker's cysts in relation to degree of joint effusion.

sponding figures for moderate and severe effusion were $38 \%(19 / 50)$ and $55.55 \%$ (5/9), respectively (Fig. 4). Logistic regression showed a positive correlation between presence of $\mathrm{BC}$ and severity of osteoarthritis and between $\mathrm{BC}$ and degree of effusion. Specifically, the Odds Ratio for presence of BC increased by about 1.42 (CI 95\%: 1.03/1.96) for each grade in the osteoarthritis severity score, whereas for each grade in the degree of joint effusion the Odds Ratio increased by about 1.82 (CI 95\%: $1.29 / 2.57)$. A correlation was also found between increasing age and prevalence of BC (Odds Ratio 1.39 CI 95\%: 1.04/1.87). Gender proved to be an independent factor with regard to the presence of $\mathrm{BC}$.

\section{DISCUSSION}

Baker's cyst forms in the popliteal region as a distension of one of the deep synovial bursae on the tendons of the gastrocnemius and semimembranosus muscles, which are often effectively continuous to form a single bursa $(1,2)$. Baker, who described these cysts more than a century ago in association with different knee disorders, postulated that they were caused by passage of synovial fluid from the joint to the bursa or by herniation of a synovial fold where the joint capsule offered less resistance (15). Studies on cadavers (16) and arthroscopy (17) or arthrography (18) with injection of contrast medium have shown this link between bursa and joint space in about 40$50 \%(16,17)$ of cysts showing that synovial fluid flows from the bursa during knee flexion, and that the inverse flow is impeded by a valve mechanism $(16,18)$. Primitive forms of distension of the gastrocnemiussemimembranosus bursa have also been documented.

Clinical examination reveals Baker's cyst as a palpable mass in the popliteal region. It may be associated with pain, pressure and difficulty in walking or in complete flexion or extension of the knee; these symptoms are often indistinguishable from those of deep venous thrombosis $(1,2)$. Baker's cyst may be mistaken if small and covered by a thick layer of tissues. On the other hand, false positives for BC may occur in the presence of lipoma, muscle disorders and less often an aneurism in the popliteal fossa.

If $\mathrm{BC}$ is correctly diagnosed it can be monitored in time. Rupture of the cyst may cause acute inflammation and functional 
failure, difficult to distinguish clinically from thrombophlebitis (pseudo-thrombophlebitic syndrome) (5). Incorrect interpretation of these symptoms can lead to inappropriate use of anticoagulants and increased risk of hemorrhage.

According to many authors and our experience, ultrasonographic examination of the joint is a valid method of diagnosing $\mathrm{BC}(6,7,10,14)$. Its sensitivity is greater than that of clinical examination (4) and its accuracy is similar to that of magnetic resonance imaging (MRI) $(6,7,10)$, demonstrating also a good agreement between operators $(19,20)$.

Ultrasonography also proves to be appropriate for studying the various signs of osteoarthritis (14, 19-22). Even without validated ultrasonographic scores, experience has been gained in applying ultrasonography to joint alterations associated with osteoarthritis, such as presence of osteophytes and thinning of trochlear cartilage $(14,20,21,23-25)$. Its sensitivity in detecting changes in joint profiles is greater than that of traditional radiography (4), and for recognition of osteophytes, ultrasound findings show good correspondence with those of MRI (22) and good agreement between operators $(23,24,26)$.

The literature shows a positive correlation between gonalgia and $\mathrm{BC}$ in many cases: the prevalence of $\mathrm{BC}$ seems greater in patients with knee pain than in healthy controls $(6,27-29)$. The incidence of BC in patients with gonalgia linked to clinical symptoms and radiographic signs of osteoarthritis in the literature varies from $27 \%$ (27) to $40 \%$ (30).

Liao et al. (31) found a $12.9 \%$ prevalence of $\mathrm{BC}$ in patients with knee pain but their population included patients with different types of disease, such as rheumatoid arthritis, serinegative spondyloarthritis and microcrystal deposit arthropathy, in whom the development of joint pain may have been related to inflammatory mechanisms. Osteoarthritis seems to be the joint pathology most often associated with BC, having a greater incidence than inflammatory diseases and microcrystal arthropathies (31). Some studies identify BC as a major determinant of joint pain in patients with osteoarthritis of the knee $(27,30)$.

On the other hand, Kornaat et al. (32) failed to find a positive association between gonalgia and $\mathrm{BC}$, even for large cysts. Other interesting studies are those of Tschirch (33) who found BC in about a fifth of subjects without joint pain, while Hayashi et al. (28) recognized BC as the most frequent cystic lesion associated with osteoarthritis of the knee of different radiological severities, but found an almost identical percentage also in asymptomatic subjects without radiological evidence of osteoarthritis.

Regarding the correlation between $\mathrm{BC}$ and osteoarthritis severity, the literature contains conflicting data. Some observations do not reveal any significant association between BC and osteoarthritis severity determined by various imaging techniques $(27,29)$, whereas Tarhan et al. (6) found a greater prevalence of $\mathrm{BC}$ in more advanced radiological stages. Hayashi et al. (28) observed an increase in prevalence of a series of cystic lesions (BC, subgastrocnemic bursitis, ganglion cysts in Hoffa's fat pad) in more advanced osteoarthritis, without finding any major increment for any of these lesions.

In our study sample of 399 patients with knee pain, 293 of whom had signs of osteoarthritis, 102 cases of BC are reported ( $25.8 \%$ of the gonalgia patients studied), whereas we lack any comparison with a subgroup without joint pain. Almost all our cases of BC (97\%) also had osteophytes and the Odds Ratio for BC increased by 1.42 for each increase in osteoarthritis severity score, demonstrating that patients with severe osteoarthritis are at much higher risk of developing BC. Only in three cases did we observe $\mathrm{BC}$ in the absence of any ultrasonographic signs of osteoarthritis.

There is more agreement in the literature about the positive association between joint effusion and knee pain $(6,29-31,34)$ and on the correlation between presence/ degree of effusion and $\mathrm{BC}(27,29)$. Of the 180 joints with $\mathrm{BC}$ observed by Liao et al. (31) simultaneous joint effusion was found in as many as 165 cases $(91.7 \%)$. Hill et al. (29) and Chatzopoulos et al. (27) observed 
a significant association between more severe effusion and $\mathrm{BC}$, but not between degree of effusion and cyst size. In our study sample, the presence and degree of join effusion were correlated with the presence of BC: $34.3 \%$ (86/251) of patients with effusion also had BC. When patients were stratified by degree of effusion, we observed a major increase in the prevalence of $\mathrm{BC}$ in relation to degree of effusion: $32 \%(61 / 191)$ of patients with minor effusion had BC, against 38\% (19/50) of those with moderate effusion and $55.5 \%$ (5/9) of those with severe effusion (Odds Ratio for presence of $\mathrm{BC}$ increased 1.82 for each increase in degree of effusion).

We underline that patients with pain or joint effusion due to inflammatory joint disease were excluded from our series, and that a substantial percentage of patients with joint effusion simultaneously had signs of moderate to advanced osteoarthritis.

The positive association between osteoarthritis and joint effusion, and the fact that only three cases of $\mathrm{BC}$ were observed in the absence of signs of osteoarthritis and 17 cases of $\mathrm{BC}$ in the absence of effusion, suggest that $\mathrm{BC}$ forms as a result of passage of fluid from the joint space, favoured by increased fluid pressure in the presence of higher grade effusion. The study could be extended to include prospective observation of patients with joint effusion to evaluate evolution in time and check for any temporal sequentiality between appearance and aggravation of joint effusion and formation of BC.

We could also consider comparison with a population without joint pain or a younger population to obtain a contrast with the prevalence of $\mathrm{BC}$ in a cohort of healthy subjects.

\section{CONCLUSION}

The results of the present study demonstrate a $25.8 \%$ prevalence of $\mathrm{BC}$ in patients with knee pain and suggest a positive association between $\mathrm{BC}$ and osteoarthritis of the knee (with an increasing trend between prevalence and severity of osteoarthritis) and between BC and presence and degree of joint effusion. Since it is not always possible to make a correct clinical diagnosis of $\mathrm{BC}$, and since recognition of $\mathrm{BC}$ is useful, among other things to reduce the risk of complications of cyst rupture, it seems worthwhile identifying a target group of patients to monitor for popliteal cysts. Joint ultrasonography is a simple, fast and reliable auxiliary to clinical examination.

Our data suggest that all patients with osteoarthritis of the knee, especially advanced disease with joint effusion, should undergo ultrasonographic screening for $\mathrm{BC}$.

Conflict of interests: the authors report no conflicts of interest in this work.

\section{REFERENCES}

1. Fritschy D, Fasel J, Imbert JC, Bianchi S, Verdonk R, Wirth CJ. The popliteal cyst. Knee Surg Sports Traumatol Arthrosc. 2006; 14: 623-8.

2. Handy JR. Popliteal cysts in adults: a review. Semin Arthritis Rheum. 2001; 31: 108-18.

3. Kellgren JH, Jeffrey M, Ball J. Atlas of standard radiographs. Vol 2. Oxford: Blackwell Scientific; 1963.

4. Kane D, Balint PV, Sturrock R. Ultrasonography is superior to clinical examination in the detection and localization of knee joint effusion in rheumatoid arthritis. J Rheumatol. 2003; 30: 966-71.

5. Drescher MJ, Smally AJ. Thrombophlebitis and pseudothrombophlebitis in the ED. Am J Emerg Med. 1997; 15: 683-5.

6. Tarhan S, Unlu Z, Goktan C. Magnetic Resonance imaging and ultrasonographic evaluation of the patients with osteoarthritis: a comparative study. Clin Rheumatol. 2003; 22: 181-8.

7. Ward EE, Jacobson JA, Fessell DP, Hayes CW, van Holsbeeck M. Sonographic detection of Baker's cysts: comparison with MR imaging. AJR Am J Roentgenol. 2001; 176: 37380.

8. Backhaus M, Burmester G-R, Gerber T, Grassi W, Machold KP, Swen WA, et al. Guidelines for musculoskeletal ultrasound in rheumatology. Ann Rheum Dis. 2001; 60: 641-9.

9. Keen HI, Wakefield RJ, Grainger AJ. Can ultrasonography improve on radiographic assessement in osteoarthritis of the hands? A comparison between detected pathology. Ann Rheum Dis. 2008; 67: 1116-20.

10. Ostergaard M, Payen MC, Gideon P, Wi- 
eslander S, Cortsen M, Lorenzen I, et al. Ultrasonography in arthritis of the knee: a comparison with MR imaging. Acta Radiologica. 1995; 36: 19-26.

11. Neogi T. Clinical significance of bone changes in osteoarthritis. Ther Adv Musculoskel Dis. 2012; 4: 259-67.

12. Wakefield RJ, Balint PV, Szkudlarek M, Filippucci E, Backhaus M, D'Agostino MA, et al Musculoskeletal ultrasound including definitions for ultrasonographic pathology. J Rheumatol. 2005; 32: 2485-7.

13. Schmidt WA, Schimdt H, Schicke B, Gromnica I. Standard reference values for musculoskeletal ultrasonography. Ann Rheum Dis. 2004; 63: 988-94.

14. Meenagh G, Filippucci E, Iagnocco A, Delle Sedie A, Riente L, Bombardieri S, et al. Ultrasound imaging for rheumatologist VIII. Ultrasound imaging in osteoarthritis. Clin Exp Rheumatol. 2007; 25: 172-5.

15. Baker WM. On the formation of the synovial cysts in the leg in connection with disease of the knee joint. St Barth Hosp Rep. 1877; 13: 245-61.

16. Rauschning W. Anatomy and function of the communication between knee joint and popliteal bursae. Ann Rheum Dis. 1980; 39: 3548.

17. Johnson LL, Van Dyk GE, Johnson CA, Bays BM, Gully SM. The popliteal bursa (Baker's cyst): an arthroscopic perspective and the epidemiology. Arthroscopy. 1977; 13: 66-72.

18. Lindgren PG, Rauschning W. Radiographic investigation of popliteal cysts. Acta Radiol Diag. 1980; 21: 657-62.

19. Scheel AK, Schmidt WA, Hermann K-GA, Bruyn GA, D'Agostino MA, Grassi W, et al. Interobserver reliability of rheumatologists performing musculoskeletal ultrasonography: results from a EULAR "Train the trainers" course. Ann Rheum Dis. 2005; 64: 1043-9.

20. Iagnocco A, Perricone C, Scirocco C, Ceccarelli $\mathrm{F}$, Modesti $\mathrm{M}$, Gattamelata $\mathrm{A}$, et al. The interobserver reliability of ultrasound in knee osteoarthritis. Rheumatology. 2012; 51: 20139.

21. Keen HI, Wakefield RJ, Conaghan PG, A systematic review of ultrasonography in osteoarthritis. Ann Rheum Dis. 2009; 68: 611-9.

22. Keen HI, Wakefield RJ, Grainger AJ, Hensor EM, Emery P, Conaghan PG. Can ultrasonography improve on radiographic assessment of osteoarthritis of the hand? A comparison between radiographic and ultrasonographic detected pathology. Ann Rheum Dis. 2008; 67: 1116-20.

23. Abraham AM, Goff I, Pearce MS, Francis RM, Birrell F. Reliability and validity of ultrasound imaging of features of knee osteoar- thritis in the community. BMC Musculoskelet Disord. 2011; 12: 70.

24. Quistgaard E, Torp-Pedersen S, Christensen $\mathrm{R}$, Bliddal H. Reproducibility and inter-reader agreement of a scoring system for ultrasound evaluation of hip osteoarthritis. Ann Rheum Dis. 2006; 65: 1613-19.

25. Kazam JK, Nazarian L, Miller TT, Sofka CM, Parker L, Adler RS. Sonographic evaluation of femoral trochlear cartilage in patients with knee pain. J Ultrasound Med. 2011; 30: 797802.

26. Keen HI, Lavie F, Weakefield RJ, D'Agostino MA, Berner Hammer H, Hensor EMA. The development of a preliminary ultrasonographic scoring system for features of hand osteoarthritis. Ann Rheum Dis. 2008; 67: 651-5.

27. Chatzopoulos D, Moralidis E, Markou P, Makris V, Arsos G. Baker's cysts in knee with chronic osteoarthritic pain: a clinical, ultrasonographic, radiographic and scintigraphic evaluation. Rheumatol Int. 2008; 29: 141-6.

28. Hayashi D, Roemer FW, Dhina Z, Kwoh CK, Hannon MJ, Moore C. Longitudinal assessment of cyst-like lesions of the knee and their relation to radiographic osteoarthritis and MRI-detected effusion and synovitis in patients with knee pain. Arthritis Res Ther. 2010; 12: R172.

29. Hill C, Gale D, Chaisson C, Skinner K, Kazis L, Gale E, et al. Knee effusions, popliteal cysts and synovial thickening: association with knee pain in osteoarthritis. J Rheumatol. 2001; 28: 6.

30. Naredo E, Cabero F, Palop MJ, Collado P, Cruz A, Crespo M. Ultrasonographic findings in knee osteoarthritis: a comparative study with clinical and radiographic assessment. Osteoarthr Cartilage. 2005; 13: 568-74.

31. Liao ST, Chiou CS, Chang CC. Pathology associated to the Baker's cysts: a muscoloskeletal ultrasound study. Clin Rheumatol 2010; 29: 1043-47.

32. Kornaat PR, Bloem JL, Ceulemans LY, Riyazi N, Rosendaal FR, Nelissen RG, et al. Osteoarthritis of the knee: association between clinical features and MR imaging findings. Radiology. 2006; 239: 811-7.

33. Tschirch FT, Shnid MR, Pfirrmann CW, Romero J, Hodler J, Zanetti M. Prevalence and size of meniscal cysts, ganglionic cysts, synovial cysts of the popliteal space, fluidfilled bursae, and other fluid collections in asymptomatic knees on MR imaging. AJR Am J Roentgenol. 2003; 180: 131-6.

34. De Miguel Mendieta E, Cobo Ibanez T, Uson Jaeger J, Bonilla Hernan G, Martin Mola E, Clinial and ultrasonographic findings related to knee pain in osteoarthritis. Osteoarthr Cartil. 2006; 14: 540-4. 